\title{
SIMULAÇÃo dA QUímiCA dA ATMOSFERA POLUÍdA POR AUTOMÓVEIS MOVIDOS A ÁlCOOL
}

\author{
A. S. Pimentel e G. Arbilla
}

Departamento de Físico-Química - Instituto de Química - Universidade Federal do Rio de Janeiro - CT - Bloco A - Cidade Universitária - 21949-900 - Rio de Janeiro - RJ

Recebido em 7/5/96; aceito em 16/10/96

\begin{abstract}
SIMULATION OF THE URBAN ATMOSPHERE CHEMISTRY POLLUTED BY ALCOHOL FUELED AUTOMOBILES. Numerical modeling studies of urban atmospheres were performed with a photochemical box submodel which incorporates primary emission rates, temperature and solar irradiance rate coefficients. Actinic flux calculations were made for an appropriate latitude for Rio de Janeiro and initial concentrations and emission rates were taken from experimental data. The relative importance of individual reactions is discussed as well as the effect of enhanced aldehyde emissions from vehicles using ethanol and ethanol-containing fuel.
\end{abstract}

Keywords: troposphere; aldehyde pollution; numerical modeling.

\section{INTRODUÇÃO}

O estudo da atmosfera e dos problemas que acontecem nela tem despertado crescente interesse nos últimos anos em diversas áreas da ciência. A exploração da atmosfera e o desenvolvimento de modelos permitiu a melhor compreensão dos fenômenos envolvidos e levou à conclusão de que as atividades humanas podem produzir mudanças significativas na composição da mesma ${ }^{1-3}$. Contudo, são muitos os pontos que ainda não estão bem esclarecidos, especialmente aqueles que são importantes só em alguns países, como as conseqüências do uso de combustíveis alternativos (por exemplo, gás natural e álcool).

A poluição do ar provocada por veículos automotores leves e pesados é um problema muito sério nos grandes centros urbanos brasileiros e estrangeiros ${ }^{4-8}$. Sendo assim, um país como o Brasil, com uma frota tão ampla e diferenciada, emite uma variedade enorme de poluentes primários ${ }^{9}$ que, por sua vez, afetam grandemente a concentração de poluentes secundários.

Com o início da utilização do álcool como combustível, o Brasil tem sofrido até hoje problemas complexos de poluição ambiental, pois possui uma frota veicular bastante diversificada, onde existem carros antigos e novos, movidos por diferentes combustíveis, e veículos pesados.

Um dos principais objetivos dos estudos na área das ciências atmosféricas é relacionar as emissões de poluentes primários em uma determinada região com as concentrações de poluentes secundários e com as mudanças das diversas espécies constituintes da troposfera. Para isto é necessário construir um modelo completo e comparar com os resultados experimentais. Este tipo de estudo, tipicamente, é realizado em duas etapas ${ }^{2}$ : primeiramente é construído um "submodelo" puramente químico que, quando possível, é avaliado com medições em uma câmara de gases onde as reações são realizadas em condições controladas, independentemente das perturbações meteorológicas; e em uma segunda etapa, é construído um "submodelo" para reproduzir as condições meteorológicas do local. De uma forma geral, um modelo completo para a troposfera poluída deve incluir três aspectos:

- As emissões dos poluentes primários para cada horário do dia e para uma determinada região.

- As condições meteorológicas do local para cada estação do

E-mail: graciela@iq.ufrj.br ano, como a temperatura, umidade relativa, ventos, difusão e movimentos de massas de ar que, junto ao tempo de vida de cada uma das espécies, irão determinar se o efeito de um determinado poluente será local, regional ou global.

- Os processos químicos.

Os processos que acontecem na atmosfera são complicados ${ }^{10,11}$ e envolvem um grande número de espécies e interações simultâneas, tornando impossível o tratamento dos mecanismos envolvidos de forma analítica. É preciso, então, resolver de forma numérica o sistema de equações diferenciais acopladas resultante do modelo proposto ${ }^{12-17^{3}}$.

$\mathrm{Na}$ resolução de um modelo completo, tridimensional (3D), para a troposfera, os processos de transporte vertical e horizontal envolvem entre 5 e $30 \%$ do tempo computacional ${ }^{13-17}$. O maior custo computacional está relacionado aos fenômenos químicos, pois o conjunto de reações elementares origina um sistema de equações diferenciais acopladas, geralmente muito rígido, que deverá ser resolvido no tempo e no espaço. Entretanto, os processos físicos de transporte não podem ser negligenciados, especialmente na descrição da troposfera, onde as emissões (naturais e antropogênicas) e a difusão contribuem significativamente para a velocidade dos processos globais. A solução de um modelo $3 \mathrm{D}$ pode ser feita de forma paralela ${ }^{13}$ ou de forma seqüencial ${ }^{17}$, resolvendo em primeiro lugar os submodelos meteorológico e de emissões e, posteriormente, os submodelos químicos (reações homogêneas, heterogêneas, formação e deposição de aerossóis). Este tipo de tratamento requer tempo computacional e espaço em memória consideráveis, especialmente no período de otimização das condições de contorno e de cada uma das partes que compõem o modelo. Geralmente a parte química do modelo precisa de uma resolução temporal e espacial muito menor. Porém, o submodelo químico pode ser resolvido e analisado isoladamente, no tempo, para determinadas condições espaciais, usando um método apropriado para equações diferenciais ordinárias em um computador pessoal. O procedimento equivale à solução de um ponto representativo da matriz de pontos que descrevem o espaço tridimensional. Sendo assim, torna-se importante realizar primeiro um estudo detalhado da química do processo e da importância relativa das reações a fim de obter um submodelo químico apropriado e o mais reduzido possível, o que implica menos tempo computacional. Nesse estudo os processos físicos podem ser descritos em forma simplificada através do chamado "modelo em caixa" que envolve, também, só equações 
diferenciais ordinárias. O submodelo otimizado é o ponto de partida para a implementação do modelo completo. Existem atualmente várias propostas para simular a química da atmosfera urbana, realizadas com o objetivo de prever os níveis de ozônio e compostos nitrogenados em diferentes condições iniciais, a fim de gerar dados e conclusões para estabelecer estratégias de controle ambiental ${ }^{17-20}$. Esses modelos são conhecidos genericamente como modelos cinéticos empíricos (EKMA), e não pretendem explicar alguns dos processos químicos relevantes da atmosfera urbana de cidades brasileiras, como as reações de aldeídos e compostos relacionados.

Um outro aspecto importante a ser considerado na modelagem da atmosfera é a mudança do fluxo solar actínico, que varia com o horário do dia e mês do ano ${ }^{21}$. Além disso, este varia com a latitude, comprimento de onda e altitude, fazendo com que cada cidade, ou seja, cada problema tenha características bem próprias. A figura 1 mostra como o ângulo zenital solar varia com o horário do dia e com mês do ano e a figura 2 mostra como varia o fluxo solar com o ângulo zenital solar e com o comprimento de onda para uma latitude de $20^{\circ} \mathrm{N}$. A

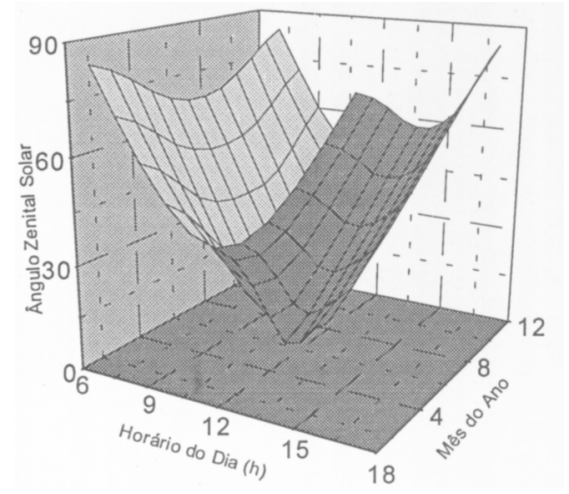

Figura 1. Variação do ângulo zenital solar com o horário do dia e mês do ano.

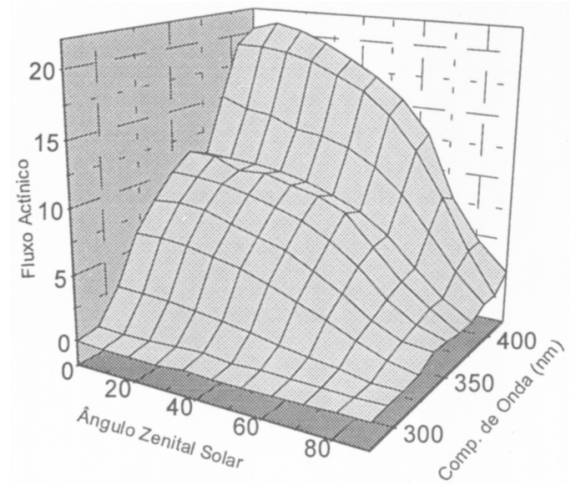

Figura 2. Variação do fluxo solar actínico (fotons $\mathrm{cm}^{-2} \mathrm{~s}^{-1}$ ) com o angulo zenital solar e comprimento de onda.

latitude de $20^{\circ} \mathrm{N}$ foi escolhida pois há uma extrema dificuldade de se obter valores deste tipo para o hemisfério $\mathrm{sul}^{2}$ e, ainda, porque a cidade do Rio de Janeiro possui latitude próxima de $20^{\circ} \mathrm{S}\left(23^{\circ} \mathrm{S}\right)$, o que equivale ao inverso da figura 1 , ou seja, ao invés de ter ângulo zenital solar próximo a $0^{\circ}$ no mês de junho, têm-se esse ângulo no mês de janeiro. Os dados representados não consideram a presença de nuvens e aerossóis, que podem diminuir sensivelmente a quantidade de energia radiante que atinge o solo. A sua influência nos processos fotoquímicos não está bem determinada e se constitui numa fonte importante de erro.

Os comprimentos de onda representados na figura 2 estão compreendidos entre 290 e $360 \mathrm{~nm}$ para uma melhor visualização da mesma, mas no cálculo foram considerados comprimentos de onda entre 290 e $600 \mathrm{~nm}$ pois existem compostos que absorvem nesta faixa de comprimento de onda. As figuras 1 e 2 apresentam resultados teóricos para o hemisfério norte ${ }^{22}$, como já foi dito anteriormente. Entretanto, alguns cálculos preliminares realizados para o hemisfério sul, utilizando o programa JSPECTRA ${ }^{23}$, demonstraram que a aproximação feita não afeta o resultado deste trabalho.

O objetivo deste trabalho é modelar as reações envolvendo aldeídos em diferentes condições, determinando os intermediários, produtos finais e a importância relativa das diferentes reações envolvidas. Visa, também, correlacionar os aldeídos com a presença de outras espécies na atmosfera tais como $\mathrm{HOx}$, $\mathrm{NO}_{\mathrm{x}}, \mathrm{O}_{3}$, nitrato de peroxiacetila (PAN), peróxidos nitrados e ácidos em geral.

\section{METODOLOGIA}

\section{Método numérico}

O método numérico e os programas de cálculo utilizados foram discutidos detalhadamente em trabalhos anteriores ${ }^{24,25}$. Por este motivo, faremos aqui só uma breve descrição da metodologia utilizada.

O modelo pode ser descrito matematicamente ${ }^{26,27}$ através de um conjunto de equações diferenciais ordinárias, acopladas, para as quais são conhecidas as condições iniciais. O sistema de equações é do tipo :

$d \boldsymbol{Y}_{i} / d t=f\left(\boldsymbol{Y}_{i}(\boldsymbol{t}), \ldots, \boldsymbol{Y}_{j}(\boldsymbol{t}), t, \boldsymbol{k}\right)$

onde $Y_{i}$ representa a espécie química $i$, e $\boldsymbol{k}$ representa o vetor das constantes de velocidade. Assim, para um mecanismo que envolve $\mathrm{N}$ espécies, resultará um sistema de $\mathrm{N}$ equações diferenciais ordinárias (ODE) acopladas, que podem ser resolvidas usando um método numérico apropriado. Para um sistema rígido, os dois métodos mais utilizados são o método de $\mathrm{Gear}^{28} \mathrm{e}$ o método Runge-Kutta-4-semi-implícito ${ }^{29}$.

Neste trabalho foram utilizados ambos os métodos. A integração das equações de velocidade foi realizada usando o programa KINETICS, desenvolvido neste laboratório. Este programa, escrito em FORTRAN 77, utiliza o método de Gear, implementado nas subrotinas LSODE e $\operatorname{VODE}^{30}$ cedidas pelo Prof. Hindmarsh (Livermoore). O programa computacional foi extensivamente discutido na referência 24. Neste trabalho, o programa foi adaptado e modificado para a inclusão da dependência das constantes de velocidade com a temperatura e fluxo solar, e para a inclusão da emissão de poluentes em função da hora do dia.

A importância relativa de cada etapa elementar e a evolução temporal de cada espécie foram calculadas usando o pacote de domínio público KINAL ${ }^{31}$, baseado no método Runge-Kutta-4semi-implícito e no método de derivadas diretas. Nesse caso, os fundamentos e metodologia do cálculo já foram discutidos com detalhe ${ }^{25}$. Note que este tipo de programa não permite a consideração de fenômenos como emissões veiculares, mudança da temperatura ou da radiação solar.

\section{DESCRIÇÃO DO MODELO}

Com o intuito de realizar uma modelagem exploratória, inicialmente foram resolvidos modelos simples, um deles envolvendo só a fotólise primária e secundária do formaldeído, um modelo mais elaborado incluindo as reações com os oxidantes $\mathrm{HO}_{\mathrm{X}}$, um modelo mais abrangente das reações do formaldeído com os oxidantes $\mathrm{HO}_{\mathrm{X}}$ e um modelo da fotodecomposição do acetaldeído envolvendo a fotólise do acetaldeído com suas principais reações. Os resultados permitiram fazer um estudo 
preliminar para compreender a química dos aldeídos, demonstrando que os sistemas de equações resultantes são muito rígidos e acoplados, e que, para explicar os fenômenos químicos na troposfera, é necessário considerar a interação entre as diversas espécies, especialmente as famílias de espécies $\mathrm{HO}_{\mathrm{x}}, \mathrm{NO}_{\mathrm{x}}$ e $\mathrm{O}_{\mathrm{x}}$.

Foi construído um modelo considerando um conjunto abrangente de espécies e reações, e a contribuição de cada um deles ao processo total foi estimada. Estes resultados permitiram eliminar mais de vinte reações elementares e algumas espécies que não contribuiram significativamente, reduzindo o tempo computacional. O modelo final é mostrado no Apêndice, e inclui 35 espécies e 55 reações químicas, que constituem um modelo bastante completo capaz de reproduzir satisfatoriamente a química da troposfera urbana. Como indicado no Apêndice, esse modelo ainda pode ser reduzido sem perda apreciável na sua capacidade de predição.

O modelo escrito em FORTRAN 77 foi implementado com um algoritmo que simula a emissão de poluentes na troposfera e outro que simula a variação do fluxo solar e temperatura com o decorrer do dia.

As expressões para os coeficientes de velocidade específica de cada reação foram extraídas da bibliografia ${ }^{2,32}$ e corrigidas, quando necessário, para a temperatura ambiente. No caso das reações fotoquímicas, o fluxo solar actínico foi calculado a partir do ângulo zenital solar de cada mês do ano e horário do dia a uma latitude de $20^{\circ} \mathrm{S}$ (próxima ao Rio de Janeiro). As concentrações iniciais e os dados de emissão de cada espécie estável foram obtidos de dados experimentais coletados da literatura ${ }^{2,6-9}$.

A determinação da velocidade de emissão de poluentes, requer um inventário de emissões para a região que está sendo modelada ${ }^{12}$. Este tipo de informação não está disponível para as cidades brasileiras, por isso foram utilizados dados estimados por Tanner et. al. ${ }^{4}$, que são consistentes com outros dados disponíveis ${ }^{9}$.

\section{RESULTADOS E DISCUSSÃO}

Inicialmente os perfis de concentrações de todas as espécies em função do tempo foram calculados utilizando as condições iniciais descritas na tabela 1 , valores médios de temperatura $\left(20^{\circ} \mathrm{C}\right.$ e $35^{\circ} \mathrm{C}$ para o inverno e verão, respectivamente), e valores de fluxo solar correspondentes às 12 horas. Estes cálculos não consideram emissões veiculares, e as condições iniciais são valores médios para a atmosfera do Rio de Janeiro, segundo os dados experimentais disponíveis ${ }^{6-8}$. As equações diferenciais foram resolvidas utilizando os programas KINETICS (LSODE e VODE) e KINAL, e os resultados são mostrados nas figuras 3-5. A concordância entre as curvas obtidas com ambos os programas é bastante boa, o que demonstra que os resultados destes métodos numéricos convergem para valores bem próximos.

Posteriormente, as concentrações de todas as espécies foram calculados em função do horário do dia, das 6 às 15 horas, usando o programa KINETICS (Figuras 6-9). A variação diurna da temperatura utilizada para o verão foi de $22^{\circ} \mathrm{C}$ a $40^{\circ} \mathrm{C}$ e para o inverno foi de $19^{\circ} \mathrm{C}$ a $27^{\circ} \mathrm{C}$. Já a variação do ângulo zenital solar para o verão foi de $2^{\circ}$ a $83^{\circ}$ e para o inverno foi de $43^{\circ}$ a $85^{\circ}$. O modelo foi resolvido com as condições iniciais e emissões veiculares mostradas na tabela 1 , que correspondem a níveis de poluição urbana da Cidade do Rio de Janeiro (Vila Izabel) obtidos da literatura ${ }^{4,5,7}$.

Foi calculada também a contribuição de cada uma das reações na velocidade total da mudança de concentração de cada uma das espécies. Estes cálculos foram feitos para uma temperatura de $20^{\circ} \mathrm{C}$ no inverno e $35^{\circ} \mathrm{C}$ no verão, com as condições iniciais da tabela 1 e coeficientes fotoquímicos que correspondem aos valores de 12 horas de um dia típico de verão e inverno, usando o programa KINAL.
Tabela 1. Concentrações iniciais e velocidades de emissão para as principais espécies utilizadas no modelo ${ }^{2,4,9}$

\begin{tabular}{|c|c|c|}
\hline espécie & $\begin{array}{l}\text { concentração inicial, } \\
\text { moléculas } / \mathrm{cm}^{3}\end{array}$ & $\begin{array}{l}\text { velocidade de emisão, } \\
\mathrm{ppb} / \mathrm{h}\end{array}$ \\
\hline $\mathrm{O}\left({ }^{3} \mathrm{P}\right)$ & $4,5010^{03}$ & - \\
\hline $\mathrm{O}\left({ }^{1} \mathrm{D}\right)$ & $4,510^{-03}$ & - \\
\hline $\mathrm{O}_{2}$ & $5,0010^{18}$ & - \\
\hline $\mathrm{O}_{3}$ & $5,0010^{10}$ & - \\
\hline $\mathrm{HO}$ & $2,5010^{06}$ & - \\
\hline $\mathrm{HO}_{2}$ & $2,0010^{08}$ & - \\
\hline NO & $1,2310^{12}$ & 12 \\
\hline $\mathrm{NO}_{2}$ & $2,4610^{11}$ & 6 \\
\hline $\mathrm{NO}_{3}$ & $2,4610^{08}$ & - \\
\hline $\mathrm{N}_{2} \mathrm{O}_{5}$ & $2,4610^{08}$ & - \\
\hline $\mathrm{HNO}_{3}$ & $9,8410^{10}$ & - \\
\hline $\mathrm{HNO}_{2}$ & $1,9710^{09}$ & - \\
\hline $\mathrm{HO}_{2} \mathrm{NO}_{2}$ & $2,4610^{08}$ & - \\
\hline $\mathrm{N}_{2} \mathrm{O}$ & $2,4610^{08}$ & - \\
\hline $\mathrm{H}_{2} \mathrm{O}$ & $5,3510^{17}$ & - \\
\hline $\mathrm{H}_{2} \mathrm{O}_{2}$ & $1,0010^{07}$ & - \\
\hline $\mathrm{CO}$ & $2,5010^{12}$ & 400 \\
\hline $\mathrm{CO}_{2}$ & $8,5010^{15}$ & - \\
\hline $\mathrm{CH}_{4}$ & $2,7510^{13}$ & - \\
\hline $\mathrm{CH}_{3} \mathrm{O}$ & $2,0010^{12}$ & - \\
\hline $\mathrm{CH}_{3} \mathrm{O}_{2}$ & $1,0010^{08}$ & - \\
\hline $\mathrm{HCHO}$ & $6,1510^{11}$ & 0,40 \\
\hline $\mathrm{CH}_{3} \mathrm{CHO}$ & $9,8410^{11}$ & 1,20 \\
\hline
\end{tabular}

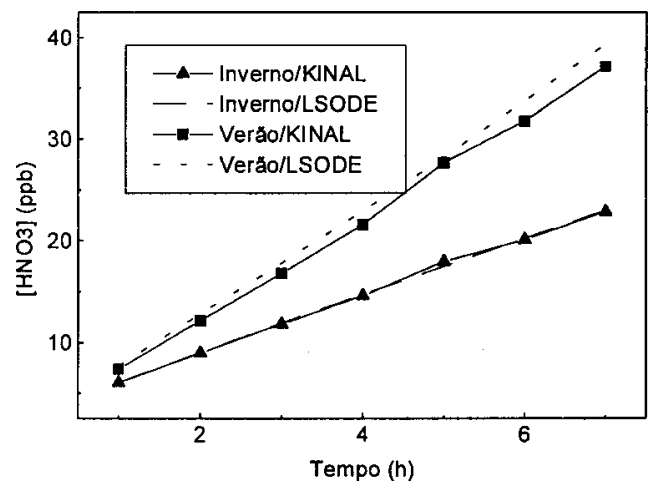

Figura 3. Modelagem da evolução temporal das concentrações de $\mathrm{HNO}_{3}$ sem emissões veiculares, com acetaldeído e formaldeído, no verão e no inverno, nas condições do modelo.

A principal via de consumo do formaldeído na atmosfera natural é a fotodecomposição. O tempo de vida desta espécie com relação a fotólise é de aproximadamente 6,3 horas no verão e 8,1 horas no inverno num dia claro. Em ambientes limpos, esta constitui a única via importante de consumo do formaldeído. Entretanto, nas condições iniciais de modelagem (atmosfera levemente poluída ou com poluição moderada), 44\% da decomposição total é causada pelas reações de HO. Note 


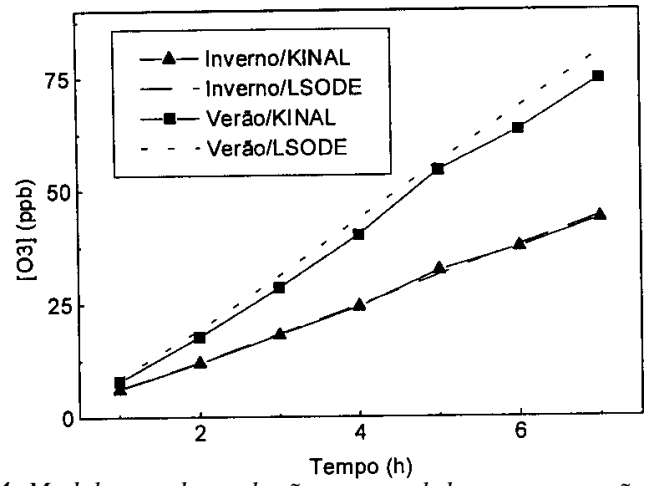

Figura 4. Modelagem da evolução temporal das concentrações de $\mathrm{O}_{3}$ sem emissões veiculares, com acetaldeído e formaldeído, no verão e no inverno, nas condições do modelo.

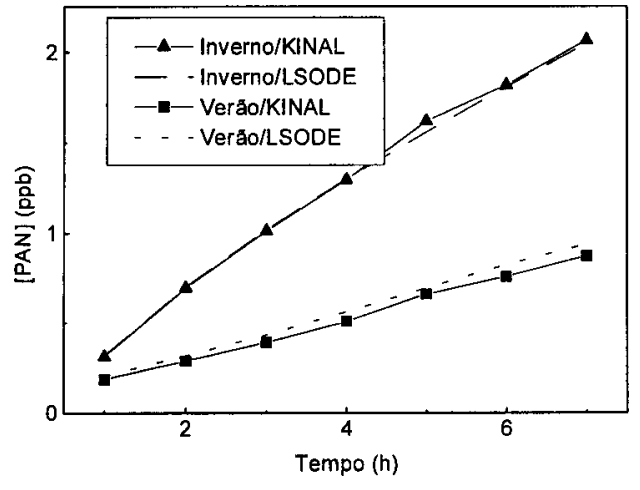

Figura 5. Modelagem da evolução temporal das concentrações de PAN sem emissões veiculares, com acetaldeído e formaldeído, no verão e no inverno, nas condições do modelo.

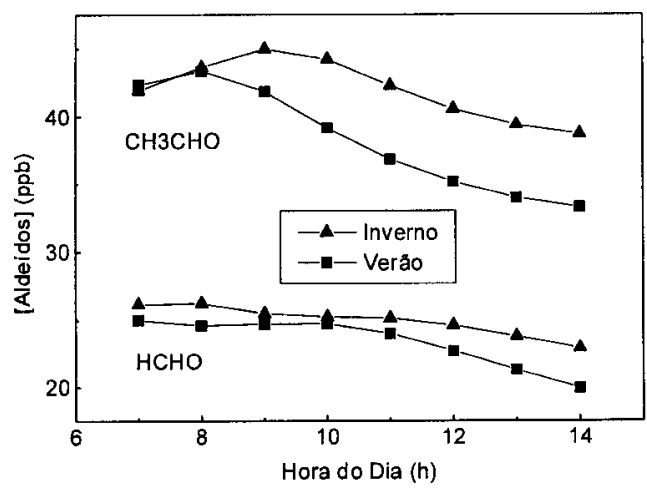

Figura 6. Concentrações de aldeídos calculadas com o modelo completo para o verão e o inverno.

que em ambientes com concentrações de acetaldeído relativamente altas, o formaldeído é um poluente secundário, formado eficientemente a partir de $\mathrm{CH}_{3} \mathrm{O}$. Já no caso do acetaldeído, a sua principal via de reação é com o $\mathrm{HO}$ para formar $\mathrm{CH}_{3} \mathrm{CO}_{3}$, precursor do PAN. O tempo de vida do acetaldeído com relação a fotólise é de aproximadamente 3,3 dias no verão e 5 dias no inverno, sendo este processo muito menos importante em dias nublados ou com pouca luminosidade. A figura 7 ilustra a relação [acetaldeído]/[formaldeído] fornecida pelo modelo e os valores superiores a 1 são típicos de ambientes urbanos com altas emissões de aldeídos.

A análise de importância das reações mostra claramente que o aumento considerável na concentração de PAN é devido às emissões veiculares de aldeídos. As concentrações calculadas

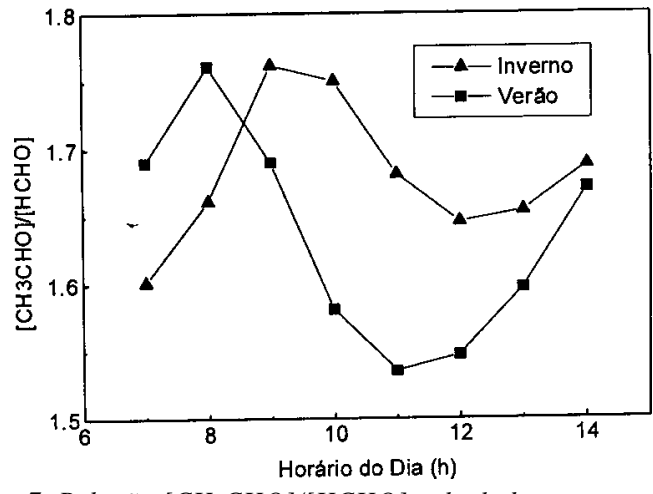

Figura 7. Relação [ $\left.\mathrm{CH}_{3} \mathrm{CHO}\right] /[\mathrm{HCHO}]$ calculada com o modelo completo para o inverno e verão.

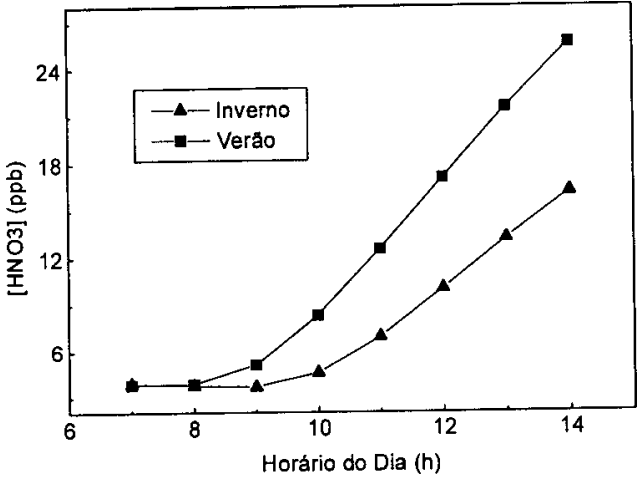

Figura 8. Concentrações de $\mathrm{HNO}_{3}$ calculadas com o modelo completo para o verão e o inverno.

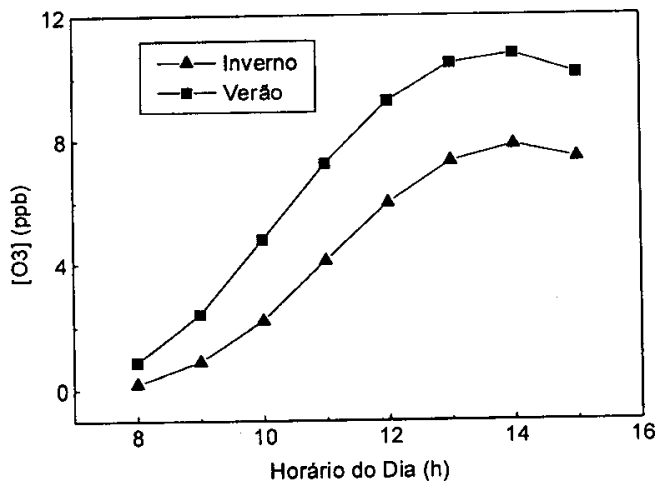

Figura 9. Concentrações de $\mathrm{O}_{3}$ calculadas com o modelo completo para o verão e o inverno.

correspondem a níveis de poluição moderada segundo os critérios de poluição aceitos por grupos de pesquisa ${ }^{2}$. O principal processo de formação do PAN é a reação 47: $\mathrm{CH}_{3} \mathrm{CO}_{3}+\mathrm{NO}_{2}=$ $\mathrm{CH}_{3} \mathrm{CO}_{3} \mathrm{NO}_{2}$, que compete com a reação 49: $\mathrm{CH}_{3} \mathrm{CO}_{3}+\mathrm{NO}$ que em atmosfera de oxigênio produz radicais $\mathrm{CH}_{3} \mathrm{O}_{2}$. O $\mathrm{CH}_{3} \mathrm{CO}_{3}$ é formado a partir da reação de acetaldeído com $\mathrm{HO}$. Pela modelagem pode-se observar que a formação de PAN aumenta bastante com o aumento da umidade relativa do ar e a diminuição da temperatura (Figura 10) e levemente com o aumento da radiação solar para uma mesma temperatura. $\mathrm{O}$ aumento da umidade relativa e da radiação solar ocasionam um acréscimo na concentração de radicais HO. Alguns cálculos preliminares para valores de umidade relativa do ar entre $30 \%$ e $100 \%$, demostraram que a concentração de PAN aumenta em torno de $40 \%$ no final do dia com o aumento da umidade nesse intervalo. Já o aumento da radiação solar, de valores típicos do 


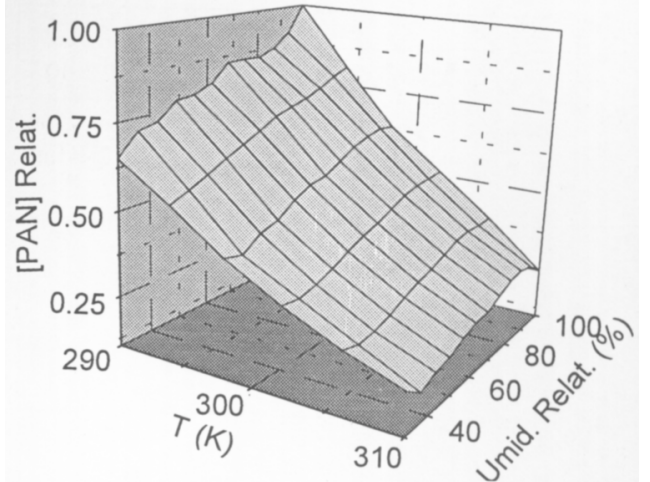

Figura 10. Concentração relativa de PAN em função da umidade relativa e temperatura. Os valores de concentração são relativos aos valores máximos para o verão.

inverno a valores típicos do verão, contribui muito pouco para um aumento na concentração de PAN. Maiores níveis de emissões de NO irão produzir uma diminuição na concentração de PAN (Figuras 11 e 12). Por outro lado, o PAN é termicamente instável e decompõe-se unimolecularmente num processo muito dependente da temperatura. $\mathrm{O}$ tempo de vida a $10^{\circ} \mathrm{C}, \tau=$ $1 / \mathrm{k}_{48}$, é de 8,6 horas, entretanto a $30^{\circ} \mathrm{C}$ é de somente $22 \mathrm{mi}$ nutos. Assim o PAN atua como um composto de reserva de $\mathrm{NO}_{2}$, ou seja, em temperaturas e/ou concentrações de $\mathrm{NO}$ baixas, a decomposição do PAN é inibida. Pelo contrário, com o aumento da temperatura ou altos níveis de NO no ar, os processos de decomposição e a reação competitiva para formar $\mathrm{CH}_{3} \mathrm{CO}_{2}$ crescem em importância e a quantidade de PAN resultante é menor. A natureza não linear do sistema torna difícil predizer o seu comportamento para um determinado conjunto de condições iniciais. Dependendo da temperatura, umidade, radiação solar e concentração dos $\mathrm{NO}_{\mathrm{x}}$, as concentrações de PAN mudarão, sendo maiores em geral para dias úmidos, frescos e com sol.

As concentrações de ácidos orgânicos, como ácido fórmico e acético, calculadas com o modelo completo, foram relativamente baixas. Porém este resultado não pode ser usado como uma indicação de um baixo índice de poluição porque o modelo não deu ênfase à descrição das reações de hidrocarbonetos e compostos orgânicos como cetona, podendo ser estas as principais vias de formação de ácidos orgânicos. Entretanto, a concentração de ácidos inorgânicos $\left(\mathrm{HNO}_{3}\right)$ apresentou-se muito grande (10-30 ppb), o que corresponde a altos níveis de poluição (Figura 8). Este resultado deve-se à lentidão do processo de decomposição fotoquímica do ácido nítrico. Por exemplo, com ângulo zenital solar de $0^{\circ}$, o tempo de vida desta espécie, calculado considerando só a decomposição fotoquímica, é de

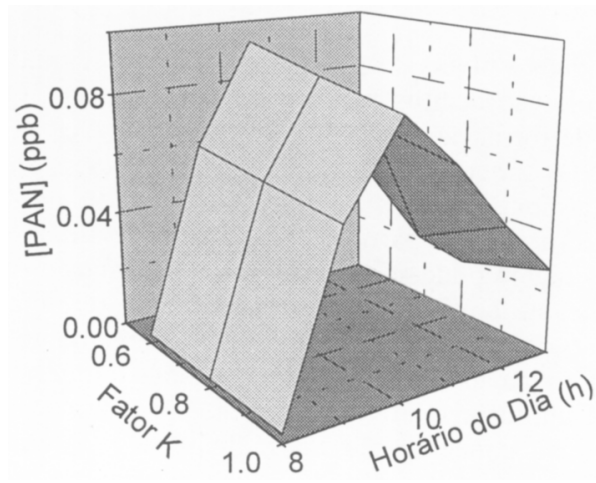

Figura 11. Concentração de PAN em função do horário do dia e concentração de $N O$ (Fator $K$ ) calculada com o modelo completo para o verão. K igual a 1.0 refere-se a uma emissão de NO de 12 ppb/h.

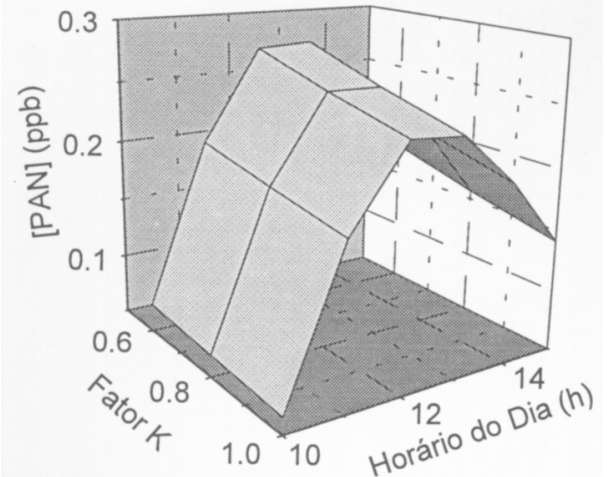

Figura 12. Concentração de PAN em função do horário do dia e concentração de $\mathrm{NO}$ (Fator $K$ ) calculada com o modelo completo para o inverno. K igual a 1.0 refere-se a uma emissão de $12 \mathrm{ppb} / \mathrm{h}$.

aproximadamente 20 dias. Contudo os processos de deposição na superfície terrestre e dissolução em água, principal motivo da chuva ácida, são relativamente importantes e contribuem para diminuir o tempo de vida do ácido na troposfera. Estes processos não foram considerados neste modelo simplificado, sendo assim os valores calculados provavelmente mais altos que os valores reais.

A figura 9 mostra que a concentração de ozônio no verão, em todos os horários do dia, é sempre maior que no inverno. Isso se deve ao balanço das reações de formação e consumo da espécie. A formação de $\mathrm{O}_{3}$ acontece principalmente pela reação 1: $\mathrm{O}\left({ }^{3} \mathrm{P}\right)+\mathrm{O}_{2} \rightarrow \mathrm{O}_{3}$ e o consumo pela reação 6: $\mathrm{NO}+\mathrm{O}_{3}$ $\rightarrow \mathrm{NO}_{2}+\mathrm{O}_{2}$. A análise de importância das reações mostra que a relação entre as contribuições das reações 1 (formação) e 6 (decomposição) é ligeiramente maior no verão do que no inverno (Tabela 2 e Figura 9). Inclusive, existem evidências experimentais $^{2}$, obtidas em alguns lugares da Europa e Estados Unidos, de que a concentração de ozônio é maior no verão do que no inverno.

\section{CONCLUSÕES}

Neste trabalho, apresentamos um submodelo puramente químico com o objetivo de correlacionar a emissão veicular de poluentes na atmosfera de centros urbanos com a formação de poluentes secundários, e as mudanças na composição do ar. $\mathrm{O}$ modelo simula também a influência da variação do fluxo solar e da temperatura nos processos químicos. Ainda que muito simples, este modelo pode explicar muitos dos aspectos essenciais dos fenômenos que ocorrem na atmosfera, e pode ser considerado como uma indicação de que os processos fotoquímicos são muito importantes na descrição da atmosfera de centros urbanos como o Rio de Janeiro, diferentemente do que acontece em outras cidades, onde os fatores topográficos e meteorológicos são os que determinam a composição atmosférica ${ }^{33}$.

A modelagem numérica da fotodecomposição dos aldeídos mostrou uma boa concordância com os dados experimentais ${ }^{4-7}$ coletados em diferentes horas do dia. A concordância é muito boa para quase todas as espécies, com exceção do $\mathrm{HNO}_{3}$. As concentrações de $\mathrm{PAN}$ e $\mathrm{O}_{3}$ apresentam um máximo para o qual a localização e intensidade dependem muito das condições atmosféricas (temperatura, umidade e radiação solar) e emissões veiculares. Para as condições predominantes do Rio de Janeiro no inverno, a concentração máxima é em torno de $0,30 \mathrm{ppb}$ para o PAN e $7 \mathrm{ppb}$ para o $\mathrm{O}_{3}$ nas primeiras horas da tarde. Estes resultados possuem uma boa concordância com os valores experimentais para a maioria dos dias monitorados ${ }^{4,34}$. Contudo, o modelo mostra que alguns episódios de maior poluição poderão ser observados em dias úmidos e mais frios que o normal, ou com condições meteorológicas especiais, como acontecido recentemente na cidade do Rio de Janeiro ${ }^{35}$. 
Tabela 2. Contribuição de cada uma das reações à velocidade (absoluta) de variação das concentrações das principais espécies no verão e inverno.

\begin{tabular}{|c|c|c|c|c|}
\hline \multirow[b]{2}{*}{ Espécie } & \multicolumn{2}{|c|}{ Janeiro } & \multicolumn{2}{|c|}{ Junho } \\
\hline & Reações & $\begin{array}{c}\text { Contribuição } \\
\text { das Reações } \\
(\%)\end{array}$ & Reações & $\begin{array}{c}\text { Contribuição } \\
\text { das Reações } \\
(\%)\end{array}$ \\
\hline \multirow[t]{3}{*}{$\mathrm{O}\left({ }^{3} \mathrm{P}\right)$} & 1 & 50,00 & 1 & 50,00 \\
\hline & 10 & 49,82 & 10 & 49,89 \\
\hline & 4 & 0,18 & 4 & 0,11 \\
\hline \multirow[t]{3}{*}{$\mathrm{O}\left({ }^{1} \mathrm{D}\right)$} & 2 & 50,0 & 2 & 52,56 \\
\hline & 4 & 44,49 & 4 & 44,55 \\
\hline & 15 & 5,50 & 15 & 0,11 \\
\hline \multirow[t]{5}{*}{$\mathrm{O}_{2}$} & 1 & 50,11 & 1 & 50,04 \\
\hline & 6 & 48,84 & 6 & 49,00 \\
\hline & 34 & 0,75 & 34 & 0,78 \\
\hline & 2 & 0,20 & 2 & 0,12 \\
\hline & 11 & 0,10 & 11 & 0,06 \\
\hline \multirow{4}{*}{$\mathrm{O}_{3}$} & 1 & 50,49 & 1 & 50,44 \\
\hline & 6 & 49,21 & 6 & 49,39 \\
\hline & 2 & 0,20 & 2 & 0,12 \\
\hline & 11 & 0,10 & 11 & 0,06 \\
\hline \multirow[t]{7}{*}{$\mathrm{HO}$} & 9 & 37,19 & 9 & 36,22 \\
\hline & 45 & 16,78 & 45 & 18,01 \\
\hline & 13 & 12,95 & 23 & 13,13 \\
\hline & 23 & 11,69 & 22 & 12,03 \\
\hline & 22 & 10,14 & 13 & 10,34 \\
\hline & 32 & 8,03 & 32 & 7,73 \\
\hline & outras & 3,19 & outras & 2,55 \\
\hline \multirow[t]{7}{*}{$\mathrm{HO}_{2}$} & 9 & 36,86 & 9 & 40,62 \\
\hline & 34 & 17,74 & 34 & 19,95 \\
\hline & 14 & 13,16 & 14 & 9,39 \\
\hline & 21 & 13,14 & 21 & 9,36 \\
\hline & 39 & 8,48 & 39 & 9,21 \\
\hline & 32 & 7,96 & 32 & 8,67 \\
\hline & outras & 2.66 & outras & 2,80 \\
\hline \multirow[t]{4}{*}{ NO } & 10 & 48,58 & 10 & 48,40 \\
\hline & 6 & 47,51 & 6 & 47,50 \\
\hline & 9 & 1,52 & 9 & 1,53 \\
\hline & outras & 2,38 & outras & 2,58 \\
\hline \multirow[t]{4}{*}{$\mathrm{NO}_{2}$} & 10 & 46,60 & 10 & 48,17 \\
\hline & 6 & 45,58 & 6 & 47,28 \\
\hline & 9 & 1,46 & 9 & 1,52 \\
\hline & outras & 3,85 & outras & 3,02 \\
\hline \multirow[t]{5}{*}{$\mathrm{NO}_{3}$} & 12 & 35,91 & 12 & 31,34 \\
\hline & 18 & 35,91 & 18 & 31,33 \\
\hline & 11 & 13,91 & 17 & 18,48 \\
\hline & 17 & 13,90 & 11 & 18,29 \\
\hline & outras & 0,38 & outras & 0,56 \\
\hline \multirow[t]{2}{*}{$\mathrm{N}_{2} \mathrm{O}_{5}$} & 12 & 50,00 & 12 & 50,01 \\
\hline & 18 & 50,00 & 18 & 49,99 \\
\hline
\end{tabular}

Continuação Tabela 2

\begin{tabular}{|c|c|c|c|c|}
\hline \multirow[b]{2}{*}{ Espécie } & \multicolumn{2}{|c|}{ Janeiro } & \multicolumn{2}{|c|}{ Junho } \\
\hline & Reações & $\begin{array}{c}\text { Contribuição } \\
\text { das Reações } \\
(\%)\end{array}$ & Reações & $\begin{array}{c}\text { Contribuição } \\
\text { das Reações } \\
(\%)\end{array}$ \\
\hline \multirow[t]{3}{*}{$\mathrm{HNO}_{3}$} & 13 & 99,32 & 13 & 99,37 \\
\hline & 55 & 0,44 & 55 & 0,37 \\
\hline & 30 & 0,24 & 30 & 0,26 \\
\hline \multirow[t]{3}{*}{$\mathrm{HNO}_{2}$} & 23 & 53,26 & 23 & 51,95 \\
\hline & 22 & 46,19 & 22 & 47,58 \\
\hline & 24 & 0,55 & 24 & 0,46 \\
\hline \multirow[t]{2}{*}{$\mathrm{HO}_{2} \mathrm{NO}_{2}$} & 14 & 50,02 & 14 & 50,09 \\
\hline & 21 & 49,98 & 21 & 49,91 \\
\hline \multirow[t]{4}{*}{$\mathrm{H}_{2} \mathrm{O}$} & 45 & 64,82 & 45 & 68,04 \\
\hline & 32 & 31,03 & 32 & 29,20 \\
\hline & 15 & 2,03 & 15 & 1,12 \\
\hline & outras & 2,13 & outras & 1,63 \\
\hline \multirow[t]{3}{*}{$\mathrm{H}_{2} \mathrm{O}_{2}$} & 19 & 52,60 & 19 & 51,26 \\
\hline & 20 & 46,78 & 20 & 48,40 \\
\hline & 16 & 0,63 & 16 & 0,35 \\
\hline \multirow[t]{5}{*}{$\mathrm{CO}$} & 32 & 38,47 & 32 & 37,59 \\
\hline & 38 & 30,97 & 38 & 33,00 \\
\hline & 39 & 20,49 & 39 & 19,98 \\
\hline & 44 & 5,35 & 44 & 4,90 \\
\hline & 25 & 4,71 & 25 & 4,54 \\
\hline \multirow[t]{2}{*}{$\mathrm{CO}_{2}$} & 49 & 94,34 & 49 & 94,65 \\
\hline & 25 & 5,66 & 25 & 5,35 \\
\hline \multirow[t]{2}{*}{$\mathrm{CH}_{3} \mathrm{O}$} & 34 & 50,00 & 34 & 50,00 \\
\hline & 33 & 50,00 & 33 & 50,00 \\
\hline \multirow[t]{4}{*}{$\mathrm{CH}_{3} \mathrm{O}_{2}$} & 33 & 50,00 & 33 & 50,00 \\
\hline & 49 & 45,83 & 49 & 46,62 \\
\hline & 44 & 3,12 & 44 & 2,83 \\
\hline & 29 & 1,05 & 29 & 0,74 \\
\hline \multirow[t]{5}{*}{$\mathrm{HCHO}$} & 34 & 48,05 & 34 & 48,14 \\
\hline & 32 & 21,57 & 32 & 20,91 \\
\hline & 38 & 17,36 & 38 & 18,35 \\
\hline & 39 & 11,49 & 39 & 11,11 \\
\hline & 41 & 1,54 & 41 & 1,49 \\
\hline \multirow[t]{2}{*}{$\mathrm{CH}_{3} \mathrm{CHO}$} & 45 & 93,76 & 45 & 94,71 \\
\hline & 44 & 6,24 & 44 & 5,29 \\
\hline \multirow[t]{2}{*}{$\mathrm{HCO}_{2} \mathrm{H}$} & 41 & 99,83 & 41 & 99,86 \\
\hline & 42 & 0,17 & 42 & 0,14 \\
\hline \multirow[t]{4}{*}{$\mathrm{CH}_{3} \mathrm{CO}_{3}$} & 45 & 42,92 & 45 & 48,27 \\
\hline & 49 & 41,95 & 49 & 44,27 \\
\hline & 47 & 8,05 & 47 & 5,73 \\
\hline & 48 & 7,08 & 48 & 1,73 \\
\hline \multirow[t]{2}{*}{ PAN } & 47 & 53,23 & 47 & 76,76 \\
\hline & 48 & 46,77 & 48 & 23,24 \\
\hline
\end{tabular}


Note-se que o fluxo solar que atinge o solo depende de outros fatores além daqueles mencionados acima, como a presença de nuvens e aerossóis, que diminui sensivelmente a quantidade de energia radiante. Neste modelo simplificado, este efeito não foi considerado, o que leva a uma maior incerteza nas determinações quantitativas.

A relação $\mathrm{CH}_{3} \mathrm{CHO} / \mathrm{HCHO}$ é uma característica fundamental dos países que utilizam álcool como combustível. Em lugares muito poluídos, como túneis com grande fluxo de veículos, esta relação pode atingir valores muito altos, em torno de 4-5. Em lugares moderadamente poluídos observam-se valores em torno de 1,5. No caso de países que não utilizam álcool como combustível esta relação é sempre menor que 1, e o formaldeído é, certamente, o aldeído mais abundante, com concentrações que superam a concentração dos outros aldeídos em uma ordem de grandeza. Em atmosferas limpas, a principal fonte de formaldeído é a sequiência de reações 29, 33 e 34. Entretanto, nas condições desta modelagem, as principais fontes de formaldeído são as emissões veiculares e a seqüência de reações 44, 45, 49, 33 e 34. Em ambos os casos acontece a reação: $\mathrm{CH}_{3} \mathrm{O}+\mathrm{O}_{2} \rightarrow \mathrm{HCHO}+\mathrm{HO}_{2}$. Em ambientes não poluídos o $\mathrm{CH}_{3} \mathrm{O}$ é um produto secundário do $\mathrm{CH}_{4}$ naturalmente presente na atmosfera. Entretanto, pelo estudo da tabela 2, pode-se deduzir que, nesta modelagem, o $\mathrm{CH}_{3} \mathrm{O}$ provém majoritariamente do $\mathrm{CH}_{3} \mathrm{CHO}$.

A análise de importância revelou que os processos de remoção, como a fotólise da água, não são importantes. Nesta análise e no cálculo dos coeficientes de sensibilidade das reações observou-se que as reações que se encontram marcadas com asterisco (Apêndice) são as menos importantes. O modelo reduzido, sem a consideração destes processos, fornece resultados igualmente satisfatórios para as principais espécies.

O etanol, principal componente do álcool combustível, provoca um aumento acentuado da emissão de aldeídos na troposfera. Por outro lado, dos resultados deste trabalho, pode-se concluir que, embora a emissão direta de poluentes por veículos movidos a álcool seja menor do que a feita por veículos movidos a gasolina, os aldeídos sofrem reações que modificam apreciavelmente as concentrações de $\mathrm{HO}_{\mathrm{x}}$ e $\mathrm{NO}_{\mathrm{x}}$ e aumentam as concentrações de oxidantes secundários e aerossóis. Certamente, a elaboração de um modelo mais completo torna-se necessária para obter resultados quantitativos de melhor qualidade, ou seja, provavelmente a concentração de ácidos orgânicos deve aumentar quando forem incluídas no modelo reações de hidrocarbonetos, radicais $\mathrm{CH}_{3} \mathrm{CO}_{2}$ e compostos oxigenados. Atualmente está sendo desenvolvido um novo modelo no qual a química do etanol e dos hidrocarbonetos voláteis é considerada de forma explícita. Também serão considerados os processos de deposição e difusão em forma mais detalhada.

Finalmente pode-se notar que do ponto de vista numérico e computacional, o método numérico que mostrou melhor desempenho para a solução deste sistema foi o Método de Gear, nas sub-rotinas LSODE e VODE, pois demandam menos tempo computacional e menor número de passos de integração para atingir convergência. Contudo os resultados são perfeitamente concordantes para qualquer uma das três sub-rotinas utilizadas, o que indica que os resultados não dependem apreciavelmente dos métodos numéricos que foram utilizados.

Apêndice. Modelo Químico para a troposfera poluída por veículos movidos a álcool. As reações indicadas com asterisco são as de menor importância tanto no verão quanto no inverno, conforme a análise de sensibilidade, e por isto podem ser eliminadas do modelo.

\begin{tabular}{|c|c|}
\hline Reações & Velocidades específicas (moléculas $/ \mathrm{cm}^{3} \mathrm{~s}$ ) \\
\hline 1) $\mathrm{O}\left({ }^{3} \mathrm{P}\right)+\mathrm{O}_{2} \rightarrow \mathrm{O}_{3}$ & $\mathrm{k}_{1}=2,78 \times 10^{-14}$ \\
\hline \multirow[t]{2}{*}{ 2) $\mathrm{O}_{3}+h v \rightarrow \mathrm{O}\left({ }^{1} \mathrm{D}\right)+\mathrm{O}_{2}$} & $\mathrm{j}_{2}($ verão $)=-2,98 \times 10^{-4}+5,94 \times 10^{-5} \mathrm{H}-2,48 \times 10^{-6} \mathrm{H}^{2}$ \\
\hline & $\mathrm{j}_{2}$ (inverno) $=-1,64 \times 10^{-4}+3,21 \times 10^{-5} \mathrm{H}-1,34 \times 10^{-6} \mathrm{H}^{2}$ \\
\hline $\left.3^{*}\right) \mathrm{O}_{3}+h v \rightarrow \mathrm{O}\left({ }^{3} \mathrm{P}\right)+\mathrm{O}_{2}$ & $\mathrm{j}_{3}=2,30 \times 10^{-8}$ \\
\hline 4) $\mathrm{O}\left({ }^{1} \mathrm{D}\right)+\mathrm{M} \rightarrow \mathrm{O}\left({ }^{3} \mathrm{P}\right)+\mathrm{M}$ & $\mathrm{k}_{4}=3,20 \times 10^{-11} \exp (67,0 / \mathrm{T})$ \\
\hline $\left.5^{*}\right) \mathrm{HO}+\mathrm{O}_{3} \rightarrow \mathrm{O}_{2}+\mathrm{HO}_{2}$ & $\mathrm{k}_{5}=1,90 \times 10^{-12} \exp (-1000,0 / \mathrm{T})$ \\
\hline 6) $\mathrm{NO}+\mathrm{O}_{3} \rightarrow \mathrm{NO}_{2}+\mathrm{O}_{2}$ & $\mathrm{k}_{6}=3,60 \times 10^{-12} \exp (-1560,0 / \mathrm{T})$ \\
\hline 7*) $\mathrm{NO}_{2}+\mathrm{O}\left({ }^{3} \mathrm{P}\right) \rightarrow \mathrm{NO}+\mathrm{O}_{2}$ & $\mathrm{k}_{7}=6,50 \times 10^{-12} \exp (120,0 / \mathrm{T})$ \\
\hline $\left.8^{*}\right) \mathrm{HO}_{2}+\mathrm{O}_{3} \rightarrow 2 \mathrm{O}_{2}+\mathrm{HO}$ & $\mathrm{k}_{8}=1,40 \times 10^{-14} \exp (-600,0 / \mathrm{T})$ \\
\hline 9) $\mathrm{HO}_{2}+\mathrm{NO} \rightarrow \mathrm{HO}+\mathrm{NO}_{2}$ & $\mathrm{k}_{9}=3,70 \times 10^{-12} \exp (240,0 / \mathrm{T})$ \\
\hline \multirow[t]{2}{*}{ 10) $\mathrm{NO}_{2}+h v \rightarrow \mathrm{NO}+\mathrm{O}\left({ }^{3} \mathrm{P}\right)$} & $\mathrm{j}_{10}($ verão $)=-9,49 \times 10^{-3}+2,31 \times 10^{-3} \mathrm{H}-9,64 \times 10^{-5} \mathrm{H}^{2}$ \\
\hline & $\mathrm{j}_{10}$ (inverno) $=-1,61 \times 10^{-2}+3,32 \times 10^{-3} \mathrm{H}-1,39 \times 10^{-4} \mathrm{H}^{2}$ \\
\hline 11) $\mathrm{NO}_{2}+\mathrm{O}_{3} \rightarrow \mathrm{NO}_{3}+\mathrm{O}_{2}$ & $\mathrm{k}_{11}=1,20 \times 10^{-13} \exp (-2450,0 / \mathrm{T})$ \\
\hline 12) $\mathrm{NO}_{3}+\mathrm{NO}_{2} \rightarrow \mathrm{N}_{2} \mathrm{O}_{5}$ & $\mathrm{k}_{12}=6,30 \times 10^{-11}$ \\
\hline 13) $\mathrm{HO}+\mathrm{NO}_{2} \rightarrow \mathrm{HNO}_{3}$ & $\mathrm{k}_{13}=1,34 \times 10^{-11}$ \\
\hline 14) $\mathrm{HO}_{2}+\mathrm{NO}_{2} \rightarrow \mathrm{HO}_{2} \mathrm{NO}_{2}$ & $\mathrm{k}_{14}=2,70 \times 10^{-12}$ \\
\hline 15) $\mathrm{O}\left({ }^{1} \mathrm{D}\right)+\mathrm{H}_{2} \mathrm{O} \rightarrow 2 \mathrm{HO}$ & $\mathrm{k}_{15}=2,30 \times 10^{-10}$ \\
\hline 16) $\mathrm{HO}_{2}+\mathrm{HO}_{2} \rightarrow \mathrm{H}_{2} \mathrm{O}_{2}+\mathrm{O}_{2}$ & $\mathrm{k}_{16}=4,50 \times 10^{-14} \exp (1200,0 / \mathrm{T})$ \\
\hline 17) $\mathrm{NO}+\mathrm{NO}_{3} \rightarrow 2 \mathrm{NO}_{2}$ & $\mathrm{k}_{17}=1,60 \times 10^{-11} \exp (150,0 / \mathrm{T})$ \\
\hline 18) $\mathrm{N}_{2} \mathrm{O}_{5}+\mathrm{M} \rightarrow \mathrm{NO}_{2}+\mathrm{NO}_{3}+\mathrm{M}$ & $\mathrm{k}_{18}=1,09 \times 10^{-11}$ \\
\hline 19) $\mathrm{H}_{2} \mathrm{O}_{2}+\mathrm{HO} \rightarrow \mathrm{H}_{2} \mathrm{O}+\mathrm{HO}_{2}$ & $\mathrm{k}_{19}=2,90 \times 10^{-12} \exp (-160,0 / \mathrm{T})$ \\
\hline \multirow[t]{2}{*}{ 20) $\mathrm{H}_{2} \mathrm{O}_{2}+h v \rightarrow 2 \mathrm{HO}$} & $\mathrm{j}_{20}($ verão $)=-1,92 \times 10^{-5}+4,09 \times 10^{-6} \mathrm{H}-1,70 \times 10^{-7} \mathrm{H}^{2}$ \\
\hline & $\mathrm{j}_{20}$ (inverno) $=-1,81 \times 10^{-5}+3,66 \times 10^{-6} \mathrm{H}-1,52 \times 10^{-7} \mathrm{H}^{2}$ \\
\hline 21) $\mathrm{HO}_{2} \mathrm{NO}_{2} \rightarrow \mathrm{HO}_{2}+\mathrm{NO}_{2}$ & $\mathrm{k}_{21}=1,30 \times 10^{14} \exp (-10418,0 / \mathrm{T})$ \\
\hline
\end{tabular}


Apêndice. Continuação

\begin{tabular}{|c|c|}
\hline Reações & Velocidades específicas (moléculas $/ \mathrm{cm}^{3} \mathrm{~s}$ ) \\
\hline 22) $\mathrm{NO}+\mathrm{HO} \rightarrow \mathrm{HONO}$ & $\mathrm{k}_{22}=1,12 \times 10^{-11}$ \\
\hline 23) $\mathrm{HONO}+h v \rightarrow \mathrm{HO}+\mathrm{NO}$ & $\begin{array}{l}\mathrm{j}_{23} \text { (verão) }=-4,35 \times 10^{-3}+1,00 \times 10^{-3} \mathrm{H}-4,18 \times 10^{-5} \mathrm{H}^{2} \\
\mathrm{j}_{23} \text { (inverno) }=-6,05 \times 10^{-3}+1,24 \times 10^{-3} \mathrm{H}-5,18 \times 10^{-5} \mathrm{H}^{2}\end{array}$ \\
\hline 24) $\mathrm{HONO}+\mathrm{HO} \rightarrow \mathrm{H}_{2} \mathrm{O}+\mathrm{NO}_{2}$ & $\mathrm{k}_{24}=4,90 \times 10^{-12}$ \\
\hline 25) $\mathrm{CO}+\mathrm{HO}+\left(\mathrm{O}_{2}\right) \rightarrow \mathrm{HO}_{2}+\mathrm{CO}_{2}$ & $\mathrm{k}_{25}=2,70 \times 10^{-13}$ \\
\hline 26) $\mathrm{NO}_{3}+h v \rightarrow \mathrm{NO}_{2}+\mathrm{O}\left({ }^{3} \mathrm{P}\right)$ & $\begin{array}{l}\mathrm{j}_{26}(\text { verão })=-3,34 \times 10^{-1}+9,61 \times 10^{-2} \mathrm{H}-4,01 \times 10^{-3} \mathrm{H}^{2} \\
\left.\mathrm{j}_{26} \text { (inverno }\right)=-8,89 \times 10^{-1}+1,87 \times 10^{-1} \mathrm{H}-7,80 \times 10^{-3} \mathrm{H}^{2}\end{array}$ \\
\hline $27 *) \mathrm{NO}_{3}+h v \rightarrow \mathrm{NO}+\mathrm{O}_{2}$ & $\mathrm{j}_{27}=2,20 \times 10^{-2}$ \\
\hline 28) $\mathrm{O}\left({ }^{1} \mathrm{D}\right)+\mathrm{N}_{2} \mathrm{O} \rightarrow 2 \mathrm{NO}$ & $\mathrm{k}_{28}=1,00 \times 10^{-08}$ \\
\hline 29) $\mathrm{HO}+\mathrm{CH}_{4}+\left(\mathrm{O}_{2}\right) \rightarrow \mathrm{H}_{2} \mathrm{O}+\mathrm{CH}_{3} \mathrm{O}_{2}$ & $\mathrm{k}_{29}=3,70 \times 10^{-12} \exp (-1820,0 / \mathrm{T})$ \\
\hline 30) $\mathrm{HO}+\mathrm{HNO}_{3} \rightarrow \mathrm{H}_{2} \mathrm{O}+\mathrm{NO}_{3}$ & $\mathrm{k}_{30}=1,50 \times 10^{-14} \exp (650,0 / \mathrm{T})$ \\
\hline $31 *) \mathrm{O}\left({ }^{1} \mathrm{D}\right)+\mathrm{CH}_{4} \rightarrow \mathrm{HCHO}+\mathrm{H}_{2}$ & $\mathrm{k}_{31}=1,50 \times 10^{-11}$ \\
\hline 32) $\mathrm{HCHO}+\mathrm{HO}+\left(\mathrm{O}_{2}\right) \rightarrow \mathrm{HO}_{2}+\mathrm{CO}+\mathrm{H}_{2} \mathrm{O}$ & $\mathrm{k}_{32}=8,80 \times 10^{-12} \exp (25,0 / \mathrm{T})$ \\
\hline 33) $\mathrm{CH}_{3} \mathrm{O}_{2}+\mathrm{NO} \rightarrow \mathrm{NO}_{2}+\mathrm{CH}_{3} \mathrm{O}$ & $\mathrm{k}_{33}=4,20 \times 10^{-12} \exp (180,0 / \mathrm{T})$ \\
\hline 34) $\mathrm{CH}_{3} \mathrm{O}+\mathrm{O}_{2} \rightarrow \mathrm{HCHO}+\mathrm{HO}_{2}$ & $\mathrm{k}_{34}=1,30 \times 10^{-13} \exp (-1350,0 / \mathrm{T})$ \\
\hline $\left.35^{*}\right) \mathrm{H}_{2} \mathrm{O}+\left(\mathrm{O}_{2}\right)+h v \rightarrow \mathrm{HO}_{2}+\mathrm{HO}$ & $\mathrm{j}_{35}=3,57 \times 10^{-14}$ \\
\hline $36 *) \mathrm{HO}_{2} \mathrm{NO}_{2}+h \mathrm{v} \rightarrow \mathrm{HO}_{2}+\mathrm{NO}_{2}$ & $\begin{array}{l}\mathrm{j}_{36}(\text { verão })=-2,08 \times 10^{-5}+4,31 \times 10^{-6} \mathrm{H}-1,80 \times 10^{-7} \mathrm{H}^{2} \\
\left.\mathrm{j}_{36} \text { (inverno }\right)=-1,64 \times 10^{-5}+3,28 \times 10^{-6} \mathrm{H}-1,37 \times 10^{-7} \mathrm{H}^{2}\end{array}$ \\
\hline $\left.37^{*}\right) \mathrm{HO}_{2} \mathrm{NO}_{2}+h \mathrm{v} \rightarrow \mathrm{HO}+\mathrm{NO}_{3}$ & $\begin{array}{l}\mathrm{j}_{37} \text { (verão) }=-1,33 \times 10^{-5}+2,76 \times 10^{-3} \mathrm{H}-1,15 \times 10^{-7} \mathrm{H}^{2} \\
\mathrm{j}_{37} \text { (inverno) }=-1,05 \times 10^{-5}+2,10 \times 10^{-6} \mathrm{H}-8,75 \times 10^{-8} \mathrm{H}^{2}\end{array}$ \\
\hline 38) $\mathrm{HCHO}+h v \rightarrow \mathrm{H}_{2}+\mathrm{CO}$ & $\begin{array}{l}\mathrm{j}_{38}(\text { verão })=-8,85 \times 10^{-5}+1,94 \times 10^{-5} \mathrm{H}-8,18 \times 10^{-7} \mathrm{H}^{2} \\
\mathrm{j}_{38} \text { (inverno) }=-9,50 \times 10^{-5}+1,93 \times 10^{-5} \mathrm{H}-8,04 \times 10^{-7} \mathrm{H}^{2}\end{array}$ \\
\hline 39) $\mathrm{HCHO}+\left(2 \mathrm{O}_{2}\right)+h v \rightarrow 2 \mathrm{HO}_{2}+\mathrm{CO}$ & $\begin{array}{l}\mathrm{j}_{39} \text { (verão) }=-6,38 \times 10^{-5}+1,36 \times 10^{-5} \mathrm{H}-5,71 \times 10^{-7} \mathrm{H}^{2} \\
\mathrm{j}_{39} \text { (inverno) }=-5,89 \times 10^{-5}+1,18 \times 10^{-5} \mathrm{H}-4,92 \times 10^{-7} \mathrm{H}^{2}\end{array}$ \\
\hline $40 *) \mathrm{N}_{2} \mathrm{O}_{5}+h v \rightarrow \mathrm{NO}_{2}+\mathrm{NO}_{3}$ & $\mathrm{j}_{40}=4,20 \times 10^{-4}$ \\
\hline 41) $\mathrm{HCHO}+\mathrm{HO}+\left(\mathrm{O}_{2}\right) \rightarrow \mathrm{HCO}_{2} \mathrm{H}+\mathrm{HO}_{2}$ & $\mathrm{k}_{41}=6,82 \times 10^{-13}$ \\
\hline 42) $\mathrm{HCHO}+\mathrm{HO}_{2} \rightarrow \mathrm{HO}+\mathrm{HCO}_{2} \mathrm{H}$ & $\mathrm{k}_{42}=2,30 \times 10^{-16}$ \\
\hline $43 *) \mathrm{HO}+\mathrm{HO} \rightarrow \mathrm{H}_{2} \mathrm{O}_{2}$ & $\mathrm{k}_{43}=4,30 \times 10^{-12}$ \\
\hline 44) $\mathrm{CH}_{3} \mathrm{CHO}+\left(2 \mathrm{O}_{2}\right)+h v \rightarrow \mathrm{CH}_{3} \mathrm{O}_{2}+\mathrm{HO}_{2}+\mathrm{CO}$ & $\begin{array}{l}\mathrm{j}_{44} \text { (verão) }=-1,52 \times 10^{-5}+3,11 \times 10^{-6} \mathrm{H}-1,30 \times 10^{-7} \mathrm{H}^{2} \\
\mathrm{j}_{44} \text { (inverno) }=-1,05 \times 10^{-5}+2,08 \times 10^{-6} \mathrm{H}-8,66 \times 10^{-8} \mathrm{H}^{2}\end{array}$ \\
\hline 45) $\mathrm{CH}_{3} \mathrm{CHO}+\mathrm{HO}+\left(\mathrm{O}_{2}\right) \rightarrow \mathrm{CH}_{3} \mathrm{CO}_{3}+\mathrm{H}_{2} \mathrm{O}$ & $\mathrm{k}_{45}=5,60 \times 10^{-12} \exp (310,0 / \mathrm{T})$ \\
\hline $\left.46^{*}\right) \mathrm{CH}_{3} \mathrm{CHO}+\mathrm{NO}_{3}+\left(\mathrm{O}_{2}\right) \rightarrow \mathrm{CH}_{3} \mathrm{CO}_{3}+\mathrm{HNO}_{3}$ & $\mathrm{k}_{46}=1,40 \times 10^{-12} \exp (-1860,0 / \mathrm{T})$ \\
\hline 47) $\mathrm{CH}_{3} \mathrm{CO}_{3}+\mathrm{NO}_{2} \rightarrow \mathrm{CH}_{3} \mathrm{CO}_{3} \mathrm{NO}_{2}$ & $\mathrm{k}_{47}=3,60 \times 10^{-12}$ \\
\hline 48) $\mathrm{CH}_{3} \mathrm{CO}_{3} \mathrm{NO}_{2} \rightarrow \mathrm{CH}_{3} \mathrm{CO}_{3}+\mathrm{NO}_{2}$ & $\mathrm{k}_{48}=1,95 \times 10^{16} \exp (-13543,0 / \mathrm{T})$ \\
\hline 49) $\mathrm{CH}_{3} \mathrm{CO}_{3}+\mathrm{NO}+\left(\mathrm{O}_{2}\right) \rightarrow \mathrm{NO}_{2}+\mathrm{CH}_{3} \mathrm{O}_{2}+\mathrm{CO}_{2}$ & $\mathrm{k}_{49}=2,00 \times 10^{-11}$ \\
\hline 50) $\mathrm{CH}_{3} \mathrm{CO}_{3}+\mathrm{HO}_{2} \rightarrow \mathrm{CH}_{3} \mathrm{CO}_{3} \mathrm{H}+\mathrm{O}_{2}$ & $\mathrm{k}_{50}=2,98 \times 10^{-12}$ \\
\hline 51) $\mathrm{CH}_{3} \mathrm{O}+\mathrm{NO}_{2} \rightarrow \mathrm{CH}_{3} \mathrm{ONO}_{2}$ & $\mathrm{k}_{51}=3,30 \times 10^{-13}$ \\
\hline $52 *) \mathrm{CH}_{3} \mathrm{O}+\mathrm{NO}_{2} \rightarrow \mathrm{HCHO}+\mathrm{HONO}$ & $\mathrm{k}_{52}=3,67 \times 10^{-14}$ \\
\hline 53) $\mathrm{CH}_{3} \mathrm{O}_{2}+\mathrm{HO}_{2} \rightarrow \mathrm{CH}_{3} \mathrm{OOH}+\mathrm{O}_{2}$ & $\mathrm{k}_{53}=1,70 \times 10^{-13} \exp (1000,0 / \mathrm{T})$ \\
\hline 54) $\mathrm{CH}_{3} \mathrm{O}_{2}+\mathrm{CH}_{3} \mathrm{CO}_{3} \rightarrow \mathrm{CH}_{3} \mathrm{CO}_{2} \mathrm{H}+\mathrm{HCHO}+\mathrm{O}_{2}$ & $\mathrm{k}_{54}=5,50 \times 10^{-12}$ \\
\hline 55) $\mathrm{HNO}_{3}+h v \rightarrow \mathrm{HO}+\mathrm{NO}_{2}$ & $\mathrm{j}_{55}($ verão $)=-3,49 \times 10^{-6}+7,11 \times 10^{-7} \mathrm{H}-2,96 \times 10^{-8} \mathrm{H}^{2}$ \\
\hline & $\mathrm{j}_{55}$ (inverno) $=-2,18 \times 10^{-6}+4,37 \times 10^{-7} \mathrm{H}-1,82 \times 10^{-8} \mathrm{H}^{2}$ \\
\hline
\end{tabular}




\section{AGRADECIMENTOS}

Os autores agradecem o apoio financeiro do CNPq, CAPES e FAPERJ, e a colaboração dos Professores Antônio H. Miguel e Jailson B. de Andrade. Agradecem também aos Prof. A. C. Hindmarsh (Livermore) e T. Turányi (Leeds) por terem cedido o LSODE/VODE e KINAL, respectivamente, para uso acadêmico e de pesquisa.

\section{REFERÊNCIAS E NOTAS}

1. Wayne, R. P.; Chemistry of Atmospheres, Clarendon Press, Oxford 1991.

2. Finlayson-Pitts, B. J.; Pitts, J. N. Jr.; Atmospheric Chemistry: Fundamentals and Experimental Techniques, John Wiley \& Sons, Chichester 1986.

3. Seinfeld, J. H.; Atmospheric Chemistry and Physics of Air Pollution, John Willey, Chichester 1986.

4. Tanner, R. L.; Miguel, A. H.; de Andrade, J. B.; Gaffney, J. S.; Streit, G. E.; Environ. Technol. 1988, 22, 1026.

5. Miguel, A. H.; Quím. Nova 1992, 15, 118.

6. Daisey, J. M.; Miguel, A. H.; de Andrade, J. B.; Pereira, P. A. P.; Tanner, R. L.; J. Air Pollut. Control Assoc. 1987, 37, 15.

7. Grosjean, D.; Miguel, A. H.; Tavares, T. M.; Atm. Environment 1990, $24 \mathrm{~B}, 101$.

8. de Andrade, J. B; de Andrade, M. V.; Pinheiro, H. L. C.; Regional Conference on Global Change, São Paulo 1995, 151.

9. Relatório do Laboratório de Emissões Veículares (LEV) do CENPES - PETROBRAS, 1995.

10. Leone, J. A.; Seinfeld, J. H.; Atmospheric Environment 1988, 19, 437.

11. de Aurelliano, J. V.; Acta Chimica Hungarica - Models in Chemistry, 1992, 129, 889.

12. Rethinking the Ozone Problem in Urban and Regional Air Pollution, editado pelo Comittee on Tropospheric Ozone Formation and Measurement, National Academy Press, Washington, D. C. 1991

13. Dabdub, D.; Seinfeld, J. H.; Atmospheric Environment 1994, 28, 1679.

14. Jang, J. C.; Jeffries, H. E.; Byun, D.; Pleim, J. E.; Atmospheric Environment 1995, 29, 3085.

15. Jang, J. C.; Jeffries, H. E.; Tonnesen, S.; Atmospheric Environment 1995, 29, 3101.

16. Informações recentes sobre o estado da arte, novos modelos e bases de dados, podem ser obtidos na INTERNET. Alguns dos endereços mais importantes são:

a) http://airsite.unc.edu/, página da Universidade da Carolina do Norte - EUA e Universidade de Leeds - Reino Unido dedicada a informação sobre química atmosférica e poluição. Possui conexão com páginas da NASA, da EPA (Environment Protection Agency, EUA) e de outras universidades.

b) http://www.agu.org/, página da American Geophysical Union, sociedade científica com a participação de 115 países nas áreas de química atmosférica, oceanografia e geofísica, e editora das principais revistas da área.

c) http://www.igbp.kva.se/, página do IGBP (International Geosphere-Biosphere Programme).

17. Chang, J. S.; Brost, R. A.; Isaken, S. A.; Madronich, S.;
Middleton, P.; Stockwell, W. R.; Walcek, C. J.; J. Geophys. Res. 1987, 92, 14681.

18. Jin, S.; Demerjian, K.; Atmospheric Environment 1993, $27 B, 371$.

19. Gery, M. W.; Whitten, G. Z.; Killus, J. P.; Dodge, M. C.; J. Geophys. Res., 94, D10, 12925.

20. The Updated OZIPR and OZIPW (OZIPR with Windows Interface). Volumes 1 and 2. User's Guide. Gery, M. W.; US EPA Order n ${ }^{-}$2D0848 NASA; North Carolina, 1995.

21. A intensidade total de radiação fora da atmosfera terrestre é a quantidade total de radiação por unidade de área normal à direção de propagação da luz. Esta quantidade tem um valor médio de $1368 \mathrm{~W} \mathrm{~m}^{-2}$. Do ponto de vista fotoquímico, é mais útil expressar o fluxo solar para um certo intervalo de comprimento de onda. Quando a radiação passa através da atmosfera, ela é modificada na sua intensidade e composição espectral pela absorção e dispersão devido a gases e partículas. Neste artigo, o termo fluxo solar actínico é usado para a quantidade de radiação que atinge a superfície terrestre por unidade de área e para intervalos de $5 \mathrm{~nm}$ de comprimento de onda. As unidades na figura 2 são fotons $\mathrm{cm}^{-2} \mathrm{~s}^{-1}$.

22. Demerjiam, K.; Schere, K. L.; Peterson, J. T.; Adv. Environ. Sci. Technol. 1980, 10, 369.

23. JSPECTRA: UNC Solar Radiation Models; Jeffries, H. E.; Relatório final para a US EPA, November 1991, North Carolina 1991.

24. Fonseca, A. de A.; Campinho, M. A.; Corrêa, S. M.; Arbilla, G.; Quím. Nova 1996, 19, 108.

25. Fonseca, A. de A.; Campinho, M. A.; Corrêa, S. M.; Arbilla, G.; Quím. Nova 1996, 19, 361.

26. Hirst, D. M.; A Computational Approach to Chemistry, Blackwell Scientific Publications, Oxford 1990.

27. Steinfeld, J. I.; Francisco, J. S.; Hase, W. L.; Chemical Kinetics and Dynamics, Prentice Hall, New Jersey 1989.

28. Gear, C. W.; Numerical Initial Value Problems in Ordinary Differential Equations, Prentice-Hall, Englewood Cliffs, New Jersey, USA 1971.

29. Kaps, P.; Rentrop, P.; Numer. Math. 1979, 33, 55.

30. Hindmarsh, A. C.; ACM Signum Newsletter 1980, 15, 10.

31. Turányi, T.; Computers Chem. 1990, 14, 253.

32. Atkinson, R.; Baulch, D. L.; Cox, R. A.; Hampson Jr., R. F.; Kerr, J. A.; Troe, J.; J. Phys. Chem. Ref. Data 1992, $21,1125$.

33. Stein, A. F.; Toselli, B.; Atmospheric Environment, no prelo.

34. Conforme relatado na Referência 4, de 2 a 5 de julho de 1985, foram observados máximos de 2-3 ppb de PAN. Contudo, na maior parte dos dias (entre 29 de junho e 8 de julho) os valores de PAN foram menores do que 0,5 ppb e muitas vezes não atingiram o limite de detecção do aparelho $(0,07 \mathrm{ppb})$. As concentrações medidas para ozônio foram $\leq 0,5 \mathrm{ppb}$.

35. Durante o inverno de 1996 foram registrados dias com alta poluição, provavelmente devido a uma combinação de fenômenos climáticos e problemas urbanos (engarrafamentos e obras). Ver, por exemplo, os artigos: "Dias de Poluição Máxima" ; Jornal do Brasil, 19 de junho de 1996 e "O Inverno da Inversão"; Jornal do Brasil; 20 de junho de 1996. 